
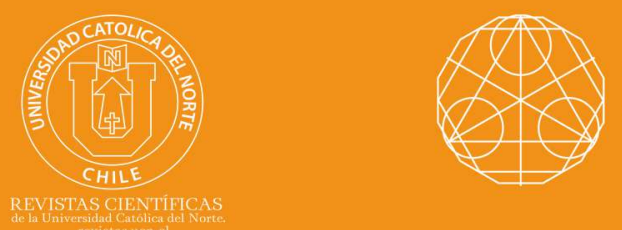

\title{
The forcing total monophonic number of a graph
}

\section{A. P. Santhakumaran ${ }^{1}$ orcid.org/0000-0002-8639-1246 \\ P. Titus ${ }^{2}$ (1) orcid.org/0000-0001-5724-0935 \\ K. Ganesamoorthy ${ }^{3}$ orcid.org/0000-0003-2769-1991 \\ M. Murugan ${ }^{4}$ [D orcid.org/0000-0002-0281-3692}

${ }^{1}$ Hindustan Institute of Technology and Science. Dept. of Mathematics, Chennai, TN, India. apskumar1953@gmail.com

${ }^{2}$ University College of Engineering Nagercoil, Dept. of Mathematics, Nagercoil, TN, India.

titusvino@yahoo.com

Coimbatore Institute of Technology, Dept. of Mathematics, Coimbatore, TN, India.

3ⓚvgm_2005@yahoo.co.in ; 4® jrfmaths@gmail.com

Received: April 2019 | Accepted: January 2021

\section{Abstract:}

For a connected graph $G=(V, E)$ of order at least two, a subset $T$ of a minimum total monophonic set $S$ of $G$ is a forcing total monophonic subset for $S$ if $S$ is the unique minimum total monophonic set containing T. A forcing total monophonic subset for $S$ of minimum cardinality is a minimum forcing total monophonic subset of $S$. The forcing total monophonic number $f_{t m}(S)$ in $G$ is the cardinality of a minimum forcing total monophonic subset of $S$. The forcing total monophonic number of $G$ is $f_{t m}(G)=\min \left\{f_{t m}(S)\right\}$, where the minimum is taken over all minimum total monophonic sets $S$ in $G$. We determine bounds for it and find the forcing total monophonic number of certain classes of graphs. It is shown that for every pair $a, b$ of positive integers with $0 \leq a<b$ and $b \geq a+4$, there exists a connected graph $G$ such that $f_{t m}(G)=a$ and $m_{t}(G)=$ $b$.

Keywords: Total monophonic set; Total monophonic number; Forcing total monophonic subset; Forcing total monophonic number.

MSC (2020): 05C12.

\section{Cite this article as (IEEE citation style):}

A. P. Santhakumaran, P. Titus, K. Ganesamoorthy, and M. Murugan,"The forcing total monophonic number of a graph", Proyecciones (Antofagasta, On line), vol. 40, no. 2, pp. 561571, 2021, doi: 10.22199/issn.0717-6279-2021-02-0031

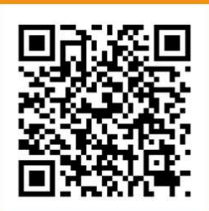

Article copyright: (C) 2021 A. P. Santhakumaran, P. Titus, K. Ganesamoorthy and M. Murugan. This is an open access article distributed under the terms of the Creative Commons License, which permits unrestricted use and distribution provided the original author and source are credited. 


\section{Introduction}

By a graph $G=(V, E)$, we mean a finite undirected connected graph without loops or multiple edges. The order and size of $G$ are denoted by $p$ and $q$ respectively. For basic graph theoretic terminology, we refer to Harary $[1,2]$. The neighborhood of a vertex $v$ is the set $N(v)$ consisting of all vertices $u$ which are adjacent with $v$. The closed neighborhood of a vertex $v$ is the set $N[v]=N(v) \bigcup\{v\}$. A vertex $v$ is an extreme vertex if the subgraph induced by its neighbors is complete.

A chord of a path $P$ is an edge joining two non-adjacent vertices of $P$. A path $P$ is called a monophonic path if it is a chordless path. A set $S$ of vertices of $G$ is a monophonic set of $G$ if each vertex $v$ of $G$ lies on a $x-y$ monophonic path for some elements $x$ and $y$ in $S$. The monophonic number of $G$ is the minimum cardinality of its monophonic sets and is denoted by $m(G)$. A vertex $v$ of a connected graph $G$ is said to be a monophonic vertex of $G$ if $v$ belongs to every minimum monophonic set of $G$. Let $S$ be a minimum monophonic set of $G$, a subset $T$ of a minimum monophonic set $S$ of $G$ is a forcing monophonic subset for $S$ if $S$ is the unique minimum monophonic set containing $T$. A forcing monophonic subset for $S$ of minimum cardinality is a minimum forcing monophonic subset of $S$. The forcing monophonic number $f_{m}(S)$ in $G$ is the cardinality of a minimum forcing monophonic subset of $S$. The forcing monophonic number of $G$ is $f_{m}(G)=\min \left\{f_{m}(S)\right\}$, where the minimum is taken over all minimum monophonic sets $S$ in $G$. The monophonic number of a graph and its variants have been studied in $[3,4,5]$. A total monophonic set of a graph $G$ is a monophonic set $S$ such that the subgraph $G[S]$ induced by $S$ has no isolated vertices. The minimum cardinality of a total monophonic set of $G$ is the total monophonic number of $G$ and is denoted by $m_{t}(G)$. The total monophonic number of a graph was studied in [6]. There are interesting applications of these concepts to the problem of designing the route for a shuttle and communication network design.

For the graph $G$ given in Figure 1.1, the minimum total monophonic sets of $G$ are $S_{1}=\{u, w, v\}$ and $S_{2}=\{u, w, x\}$ so that the total monophonic number of $G$ is $m_{t}(G)=3$. 


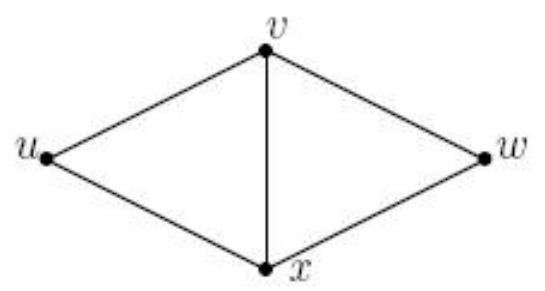

Figure 1.1: $G$

A connected graph $G$ may contain more than one minimum total monophonic set. For example, the graph $G$ given in Figure.1.1 contains two minimum total monophonic sets. For each minimum total monophonic set $S$ in $G$ there is always some subset $T$ of $S$ that uniquely determines $S$ as the minimum total monophonic set containing $T$. This motivated to introduce and investigate the concept "forcing total monophonic subsets".

The following theorems will be used in the sequel.

Theorem 1.1. [5] Each extreme vertex of a connected graph $G$ belongs to every monophonic set of $G$.

Theorem 1.2. [3] Let $G$ be a connected graph and let $S$ be the set of all monophonic vertices of $G$. Then $f_{m}(G) \leq m(G)-|M|$.

Theorem 1.3. [6] All extreme vertices and all support vertices of a connected graph $G$ belong to every total monophonic set of $G$.

Theorem 1.4. [6] For the complete graph $K_{p}(p \geq 2), m_{t}\left(K_{p}\right)=p$.

Theorem 1.5. [6] For any non-trivial tree $T$, the set of all endvertices and support vertices of $T$ is the unique minimum total monophonic set of $T$.

Theorem 1.6. [6] For any connected graph $G, m_{t}(G)=2$ if and only if $G=K_{2}$.

Throught this paper $G$ denotes a connected graph with at least two vertices. 


\section{Forcing total monophonic number}

Definition 2.1. Let $G$ be a connected graph and let $S$ be a minimum total monophonic set of $G$. A subset $T$ of a minimum total monophonic set $S$ of $G$ is a forcing total monophonic subset for $S$ if $S$ is the unique minimum total monophonic set containing $T$. A forcing total monophonic subset for $S$ of minimum cardinality is a minimum forcing total monophonic subset of $S$. The forcing total monophonic number $f_{t m}(S)$ in $G$ is the cardinality of a minimum forcing total monophonic subset of $S$. The forcing total monophonic number of $G$ is $f_{t m}(G)=\min \left\{f_{t m}(S)\right\}$, where the minimum is taken over all minimum total monophonic sets $S$ in $G$.

Example 2.2. For the graph $G$ given in Figure 1.1, $S_{1}=\{u, w, v\}$ and $S_{2}=\{u, w, x\}$ are the minimum total monophonic sets of $G$. It is clear that $f_{t m}\left(S_{1}\right)=1$ and $f_{t m}\left(S_{2}\right)=1$ so that $f_{t m}(G)=1$. By Theorem 1.5, for any non-trivial tree $T$, the set of all endvertices and support vertices of $T$ is the unique minimum total monophonic set of $T$ and so $f_{t m}(T)=0$.

The next result follows immediately from the definition of the total monophonic number and forcing total monophonic number of a graph $G$.

Result 2.3. For a connected graph $G, 0 \leq f_{t m}(G) \leq m_{t}(G) \leq p$.

Remark 2.4. The bounds in Result 2.3 are sharp. By Theorem 1.5, for any non-trivial tree $T$, the set of all endvertices and support vertices of $T$ is the unique minimum total monophonic set of $T$ and so $f_{t m}(T)=0$. By Theorem 1.4, for the complete graph $K_{p}(p \geq 2), m_{t}\left(K_{p}\right)=p$. The inequalities in Result 2.3 can be strict. For the graph $G$ given in Figure 1.1, $m_{t}(G)=3$ and $f_{t m}(G)=1$. Thus $0<f_{t m}(G)<m_{t}(G)<p$.

The following theorem is an easy consequence of the definitions of the total monophonic number and forcing total monophonic number. In fact, the theorem characterizes graphs $G$ for which the lower bound in Result 2.3 is attained and also graphs $G$ for which $f_{t m}(G)=1$ and $f_{t m}(G)=m_{t}(G)$.

Theorem 2.5. Let $G$ be a connected graph . Then

(i) $f_{t m}(G)=0$ if and only if $G$ has a unique minimum total monophonic set.

(ii) $f_{t m}(G)=1$ if and only if $G$ has at least two minimum total monophonic sets, one of which is a unique minimum total monophonic set containing one of its elements, and 
(iii) $f_{t m}(G)=m_{t}(G)$ if and only if no minimum total monophonic set of $G$ is the unique minimum total monophonic set containing any of its proper subsets.

Definition 2.6. A vertex $v$ of a connected graph $G$ is said to be a total monophonic vertex of $G$ if $v$ belongs to every minimum total monophonic set of $G$.

We observe that if $G$ has a unique minimum total monophonic set $S$, then every vertex in $S$ is a total monophonic vertex of $G$. Also, if $x$ is an extreme vertex or support vertex of $G$, then $x$ is a total monophonic vertex of $G$. For the graph $G$ given in Figure 1.1, $u$ and $w$ are the total monophonic vertices of $G$.

The next theorem and corollary are immediate consequence of the definitions of total monophonic vertex and forcing total monophonic subset of G.

Theorem 2.7. Let $G$ be a connected graph and let $\Psi_{t m}$ be the set of relative complements of the minimum forcing total monophonic subsets in their respective minimum total monophonic sets in $G$. Then $\bigcap_{F \in \Psi_{t m}} F$ is the set of total monophonic vertices of $G$.

Corollary 2.8. Let $G$ be a connected graph and let $S$ be a minimum total monophonic set of $G$. Then no total monophonic vertex of $G$ belongs to any minimum forcing total monophonic subset of $S$.

Theorem 2.9. Let $G$ be a connected graph and let $M$ be the set of all total monophonic vertices of $G$. Then $f_{t m}(G) \leq m_{t}(G)-|M|$.

Proof. Let $S$ be any minimum total monophonic set of $G$. Then $m_{t}(G)=|S|, M \subseteq S$ and $S$ is the unique minimum total monophonic set containing $S-M$. Thus $f_{t m}(G) \leq|S-M|=|S|-|M|=m_{t}(G)-|M|$.

Corollary 2.10. If $G$ is a connected graph with $m$ extreme vertices and $n$ support vertices, then $f_{t m}(G) \leq m_{t}(G)-(m+n)$.

Remark 2.11. The bound in Theorem 2.9 is sharp. For the graph $G$ given in Figure 1.1, $m_{t}(G)=3$ and $f_{t m}(G)=1$. Also, $M=\{u, w\}$ is the set of all total monophonic vertices of $G$ and so $f_{t m}(G)=m_{t}(G)-|M|$. Also the inequality in Theorem 2.9 can be strict. For the graph $G$ given in 
Figure 2.1, the minimum total monophonic sets of $G$ are $S_{1}=\{v, u, y\}$ and $S_{2}=\{v, w, x\}$ and so $m_{t}(G)=3$. It is clear that $f_{t m}\left(S_{1}\right)=1$ and $f_{\text {tm }}\left(S_{2}\right)=1$ so that $f_{\text {tm }}(G)=1$. Also, the vertex $v$ is only total monophonic vertex of $G$, we have $f_{t m}(G)<m_{t}(G)-|M|$.

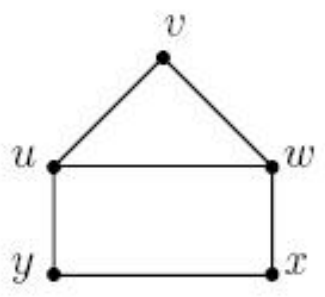

Figure 2.1: $G$

Theorem 2.12. If $G$ is a connected graph with $m_{t}(G)=2$, then $f_{t m}(G)=$ 0 .

Proof. If $m_{t}(G)=2$ then by Theorem 1.6, we have $G=K_{2}$. Thus $V(G)$ is the unique minimum total monophonic set of $G$. Also, by Theorem 2.5(i), $f_{t m}(G)=0$.

Remark 2.13. The converse of Theorem 2.12 need not be true. For the path $P_{4}$ of order 4 , the vertex set $V\left(P_{4}\right)$ is the unique minimum total monophonic set of $G$ and by Theorem 2.5(i), we have $f_{\text {tm }}\left(P_{4}\right)=0$. But the total monophonic number of $P_{4}$ is 4 .

\section{Forcing total monophonic number of some standard graphs}

Now, we proceed to determine the forcing total monophonic number of certain classes of graphs.

Theorem 3.1. For any cycle $C_{n}(n \geq 3)$,

$$
f_{t m}\left(C_{n}\right)= \begin{cases}0 & \text { if } n=3 \\ 3 & \text { if } n=4 \\ 2 & \text { if } n \geq 5\end{cases}
$$


Proof. Let $C_{n}: v_{1}, v_{2}, \ldots, v_{n}, v_{1}$ be a cycle of order $n$. We prove this theorem by considering two cases.

Case (i) $n=3$. Since $C_{3}$ is the complete graph of order 3 , by Theorems 1.4 and $2.5(\mathrm{i}), f_{t m}\left(C_{3}\right)=0$.

Case (ii) $n \geq 4$. It is clear that no 2-element subset of $V\left(C_{n}\right)$ is a total monophonic set of $C_{n}$. It is easy to verify that any minimum total monophonic sets of $C_{n}$ consists of three consecutive vertices of $C_{n}$ so that $m_{t}\left(C_{n}\right)=3$. For $n=4$, it is clear that, no minimum total monophonic set of $C_{4}$ is the unique minimum total monophonic set containing any of its proper subsets. Thus by Theorem 2.5 (iii), we have $f_{t m}\left(C_{4}\right)=3$. For $n \geq 5$, it is clear that the two non-adjacent vertices of any minimum total monophonic set $S$ of $G$ is a minimum forcing total monophonic subset of $S$ and so $f_{t m}(S)=2$. Hence $f_{t m}\left(C_{n}\right)=2$.

Theorem 3.2. For any complete graph $G=K_{p}(p \geq 2)$ or any non-trivial tree $G=T, f_{t m}(G)=0$.

Proof. Let $G=K_{p}$. By Theorem 1.4, the set of all vertices of $G$ is the unique minimum total monophonic set of $G$ and so by Theorem 2.5 (i), $f_{t m}(G)=0$. If $G$ is a non-trivial tree, then by Theorem 1.5 , the set of all endvertices and support vertices of $G$ is the unique minimum total monophonic set of $\mathrm{G}$ and so by Theorem 2.5 (i), $f_{t m}(G)=0$.

Theorem 3.3. For the complete bipartite graph $G=K_{m, n}(2 \leq m \leq n)$,

$$
f_{t m}(G)= \begin{cases}1 & \text { if } 2=m<n \\ 3 & \text { if } 2=m=n \\ 4 & \text { if } 3 \leq m \leq n\end{cases}
$$

Proof. Let $U=\left\{u_{1}, u_{2}, \ldots, u_{m}\right\}$ and $W=\left\{w_{1}, w_{2}, \ldots, w_{n}\right\}$ be the partite sets of $G$, where $m \leq n$. We prove this theorem by considering four cases.

Case 1. $2=m<n$. For any $j(1 \leq j \leq n), S_{j}=U \cup\left\{w_{j}\right\}$ is a minimum total monophonic set of $G$. Since $n \geq 3$, then by Theorem 2.5(ii), we have $f_{t m}(G)=1$.

Case 2. $2=m=n$. Since $G$ is a cycle of order 4 , the result follows from Theorem 3.1. 
Case 3. If $3=m=n$, then any minimum total monophonic set of $G$ is of the following forms: $(i) U \cup\left\{w_{j}\right\}$ for some $j(1 \leq j \leq n)$, (ii) $W \cup\left\{u_{i}\right\}$ for some $i(1 \leq i \leq m)$, or (iii) the minimum total monophonic set of $G$ formed by choosing any two elements from $U$ as well as $W$. If $3=m<n$, then any minimum total monophonic set of $G$ is either $U \cup\left\{w_{j}\right\}$ for some $j(1 \leq j \leq n)$, or the minimum total monophonic set of $G$ formed by choosing any two elements from $U$ as well as $W$. Hence in both cases, we have $m_{t}(G)=4$. Clearly, no minimum total monophonic set of $G$ is the unique minimum total monophonic set containing any of its proper subsets. Then by Theorem 2.5(iii), we have $f_{t m}(G)=m_{t}(G)=4$.

Case 4. $4 \leq m \leq n$. Then any minimum total monophonic set is formed by choosing any two elements from $U$ as well as $W$, and $G$ has at least two minimum total monophonic sets. Hence $m_{t}(G)=4$. Clearly, no minimum total monophonic set of $G$ is the unique minimum total monophonic set containing any of its proper subsets. Then by Theorem 2.5(iii), we have $f_{t m}(G)=m_{t}(G)=4$.

Theorem 3.4. For every pair $a, b$ of positive integers with $0 \leq a<b$ and $b \geq a+4$, there exists a connected graph $G$ such that $f_{\text {tm }}(G)=a$ and $m_{t}(G)=b$.

Proof. If $a=0$, let $G=K_{1, b-1}$. Then by Theorem $3.2, f_{t m}(G)=0$ and by Theorem 1.5, $m_{t}(G)=b$. Now, assume that $0<a<b$. Let $H$ be the graph formed by identifying the vertex $w$ of the path $P_{3}$ : $u, v, w$ with the central vertex $x$ of the star $K_{1, b-a-3}$, where $V\left(K_{1, b-a-3}\right)=$ $\left\{x, z_{1}, z_{2}, \cdots, z_{b-a-3}\right\}$. Let $P_{i}: x_{i}, y_{i}(1 \leq i \leq a)$ be 'a' copies of the path of order 2. The graph $G$ is obtained from $H$ and $P_{i}(1 \leq i \leq a)$ by joining each $x_{i}$ of $P_{i}$ to the vertex of $v$ of $H$ and joining each $y_{i}$ of $P_{i}$ to the vertex of $w$ of $H$. The graph $G$ is shown in Figure 3.1. Let $S=\left\{z_{1}, z_{2}, \cdots, z_{b-a-3}, u, v, w\right\}$ be the set of all endvertices and support vertices of $G$. By Theorem 1.3, every total monophonic set of $G$ contains $S$. It is clear that $S$ is not a total monophonic set of $G$. We observe that every minimum total monophonic set of $G$ contains exactly one vertex from the set $\left\{x_{i}, y_{i}\right\}$ for every $i(1 \leq i \leq a)$. Thus $m_{t}(G) \geq b$. Since $S_{1}=S \cup\left\{x_{1}, x_{2}, \cdots, x_{a}\right\}$ is a total monophonic set of $G$, it follows that $m_{t}(G)=b$. 


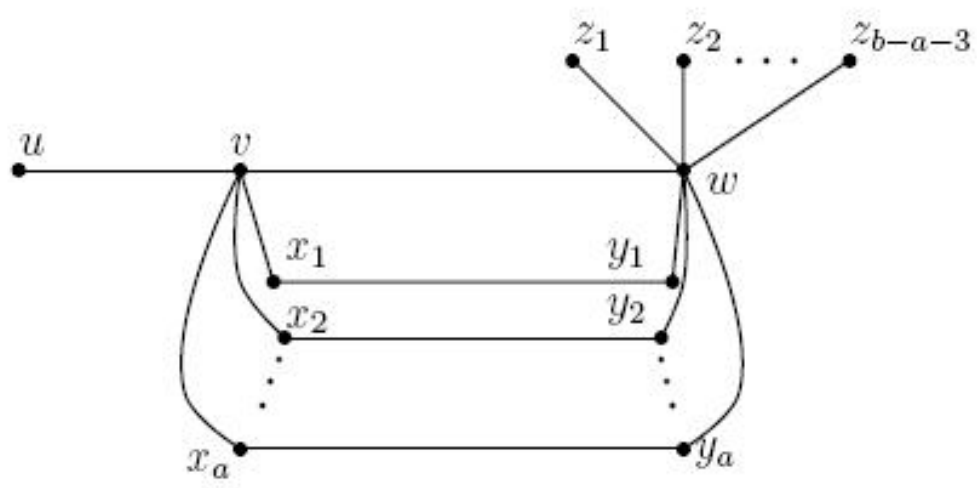

Figure 3.1: $G$

Next, we show that $f_{t m}(G)=a$. Since every minimum total monophonic set of $G$ contains $S$, it follows from Theorem 2.9 that $f_{t m}(G) \leq$ $m_{t}(G)-|S|=b-(b-a)=a$. Now, since $m_{t}(G)=b$ and every minimum total monophonic set of $G$ contains $S$, it is clear that every minimum total monophonic set $S^{\prime}$ of $G$ is of the form $S \cup\left\{u_{1}, u_{2}, \cdots, u_{a}\right\}$, where $u_{i} \in\left\{x_{i}, y_{i}\right\}$ for every $i(1 \leq i \leq a)$. Let $T$ be any proper subset of $S^{\prime}$ with $|T|<a$. Then there is a vertex $x \in S^{\prime}-S$ such that $x \notin T$. If $x=x_{i}(1 \leq i \leq a)$, then $S^{\prime \prime}=\left(S^{\prime}-\left\{x_{i}\right\}\right) \cup\left\{y_{i}\right\}$ is a minimum total monophonic set containing $T$. Similarly, if $x=y_{j}(1 \leq j \leq a)$, then $S^{\prime \prime \prime}=\left(S^{\prime}-\left\{y_{j}\right\}\right) \cup\left\{x_{j}\right\}$ is a minimum total monophonic set containing $T$. Thus $S^{\prime}$ is not the unique minimum total monophonic set containing $T$ and so $T$ is not a forcing subset of $S^{\prime}$. This is true for all minimum total monophonic sets of $G$ and so $f_{t m}(G)=a$.

Theorem 3.5. For any two positive integers $a, b$ with $1 \leq a<b$ and $b=$ $2 a$, there exists a connected graph $G$ such that $f_{m}(G)=a$ and $f_{t m}(G)=b$.

Proof. Let $C_{i}: x_{i}, y_{i}, z_{i}, u_{i}, v_{i}, x_{i}(1 \leq i \leq a)$ be " $a$ " copies of the cycle $C_{i}$ of order 5 . Let $H$ be the graph obtained from $C_{i}$ by identifying the vertices $x_{i}(1 \leq i \leq a)$, say $x$ be identified vertex. Add a new vertex $y$ to $H$, and join $y$ to $x$, thereby producing the graph $G$ shown in Figure 3.2. Since $y$ is the only extreme vertex of $G$, by Theorem 1.1, every monophonic set of $G$ contains $y$. It is observed that any monophonic set of $G$ contains exactly one vertex from each set $\left\{u_{i}, z_{i}\right\}(1 \leq i \leq a)$ so that $m(G) \geq a+1$. Since 
$S_{1}=\left\{u_{1}, u_{2}, \cdots, u_{a}, y\right\}$ is a monophonic set of $G$, it follows that $m(G)=$ $a+1$. Next, we show that $f_{m}(G)=a$. Since $y$ is the only monophonic vertex of $G$, it follows from Theorem 1.2 that $f_{m}(G) \leq m(G)-|\{y\}|=a$. It is easily seen that every minimum monophonic set $S^{\prime}$ of $G$ is of the form $\left\{m_{1}, m_{2}, \cdots, m_{a}, y\right\}$, where $m_{i} \in\left\{u_{i}, z_{i}\right\}$ for every $i(1 \leq i \leq a)$. Let $T$ be any proper subset of $S^{\prime}$ with $|T|<a$. Then there is a vertex $u \in S^{\prime}-\{y\}$ such that $u \notin T$. If $u=u_{i}(1 \leq i \leq a)$, then $S^{\prime \prime}=\left(S^{\prime}-\left\{u_{i}\right\}\right) \cup\left\{z_{i}\right\}$ is a minimum monophonic set containing $T$. Similarly, if $u=z_{j}(1 \leq j \leq a)$, then $S^{\prime \prime \prime}=\left(S^{\prime}-\left\{z_{j}\right\}\right) \cup\left\{u_{j}\right\}$ is a minimum monophonic set containing $T$. Thus $S^{\prime}$ is not the unique minimum monophonic set containing $T$ and so $T$ is not a forcing subset of $S^{\prime}$. This is true for all minimum monophonic sets of $G$ and so $f_{m}(G)=a$.

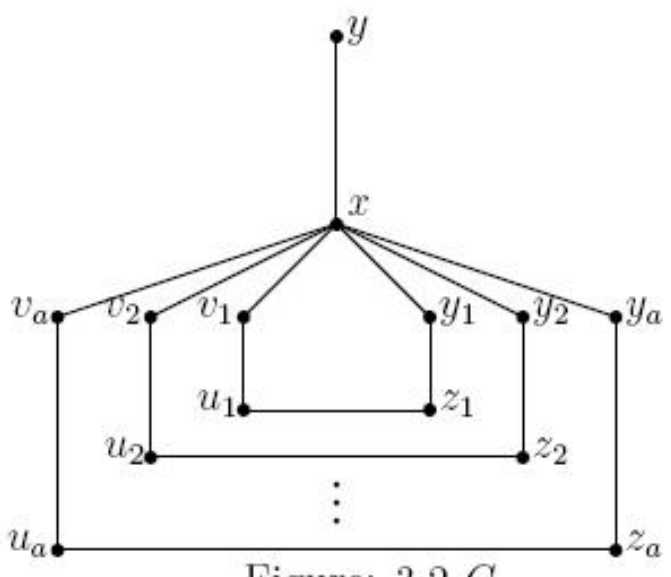

Figure: $3.2 G$

By Theorem 1.3, every minimum total monophonic set of $G$ contains $M=\{y, x\}$. Clearly, $M$ is not a total monophonic set of $G$. It is observed that any minimum total monophonic set of $G$ contains just the two vertices of any of the set from the collection of sets $\left\{\left\{u_{i}, z_{i}\right\},\left\{u_{i}, v_{i}\right\},\left\{y_{i}, z_{i}\right\},\left\{v_{i}, y_{i}\right\}\right\}$ for every $i(1 \leq i \leq a)$ so that $m_{t}(G) \geq 2 a+2$. Since $S_{2}=M \cup$ $\left\{u_{1}, u_{2}, \cdots, u_{a}, v_{1}, v_{2}, \cdots, v_{a}\right\}$ is a total monophonic set of $G$, it follows that $m_{t}(G)=2 a+2$. Since $x$ and $y$ are the only total monophonic vertices of $G$, it follows from Theorem 2.9 that $f_{t m}(G) \leq m_{t}(G)-|M|=2 a+2-2=2 a$. Now, since $m_{t}(G)=2 a+2$ and every minimum total monophonic set of $G$ contains $M$, it is easily seen that every minimum total monophonic set $S_{1}^{\prime}$ of $G$ is of the form $M \cup\left\{m_{1}, m_{1}^{\prime}, m_{2}, m_{2}^{\prime}, \ldots, m_{a}, m_{a}^{\prime}\right\}$, where both $m_{i}, m_{i}^{\prime}$ belong to just one of the sets from $\left\{\left\{u_{i}, z_{i}\right\},\left\{u_{i}, v_{i}\right\},\left\{y_{i}, z_{i}\right\},\left\{v_{i}, y_{i}\right\}\right\}$ for every $i(1 \leq i \leq a)$. Let $T^{\prime}$ be any proper subset of $S_{1}^{\prime}$ with $\left|T^{\prime}\right|<2 a$. Then there 
is a vertex $u \in S_{1}^{\prime}-M$ such that $u \notin T^{\prime}$. If $u=u_{i}$ and $u=u_{i}(1 \leq i \leq a)$ is adjacent to $z_{i}$ or adjacent to $v_{i}$, then $S_{11}=\left(S_{1}^{\prime}-\left\{u_{i}\right\}\right) \cup\left\{y_{i}\right\}$ is a minimum total monophonic set containing $T^{\prime}$. If $u=z_{i}$ and $u=z_{i}(1 \leq i \leq a)$ is adjacent to $y_{i}$ or adjacent to $u_{i}$, then $S_{12}=\left(S_{1}^{\prime}-\left\{z_{i}\right\}\right) \cup\left\{v_{i}\right\}$ is a minimum total monophonic set containing $T^{\prime}$. If $u=y_{i}(1 \leq i \leq a)$, then $S_{13}=\left(S_{1}^{\prime}-\left\{y_{i}\right\}\right) \cup\left\{u_{i}\right\}$ is a minimum total monophonic set containing $T^{\prime}$. If $u=v_{i}(1 \leq i \leq a)$, then $S_{14}=\left(S_{1}^{\prime}-\left\{v_{i}\right\}\right) \cup\left\{z_{i}\right\}$ is a minimum total monophonic set containing $T^{\prime}$. Thus $S_{1}^{\prime}$ is not the unique minimum total monophonic set containing $T^{\prime}$ and so $T^{\prime}$ is not a forcing subset of $S_{1}^{\prime}$. This is true for all minimum total monophonic sets of $G$ and so $f_{t m}(G)=2 a=b$.

\section{Acknowledgements}

The third author acknowledges support from Research supported by NBHM Project No. NBHM/ R.P.29/2015/ Fresh/ 157.

\section{References}

[1] F. Buckley and F. Harary, Distance in graphs. Redwood City, CA: Addison-Wesley, 1990.

[2] F. Harary, Graph theory. Reading, MA: Addison-Wesley, 1969. [On line]. Available: https:/ / bit.ly/ 38wIHRk

[3] K. Ganesamoorthy, "A study of monophonic number and its variants", Ph.D. thesis, Anna University, Chennai, 2013.

[4] E. M. Paluga and S. R. Canoy, "Monophonic numbers of the join and composition of connected graphs", Discrete mathematics, vol. 307, no. 9-10, pp. 1146-1154, 2007, doi: 10.1016/ j.disc.2006.08.002

[5] A. P. Santhakumaran, P. Titus and K. Ganesamoorthy, "On the monophonic number of a graph", Journal of applied mathematics \& informatics, vol. 32, no. 1-2, pp. 255-266, 2014, doi: 10.14317/jami.2014.255

[6] A. P. Santhakumaran, P. Titus, K. Ganesamoorthy, and M. Murugan, "The Total Monophonic Number of a Graph", in Proceedings of International Conference on Discrete Mathematics and its Applications to Network Science (ICDMANS 2018), Under process. 